\title{
Beyond Distraction: Virtual Reality Graded Exposure Therapy as Treatment for Pain-Related Fear and Disability in Chronic Pain
}

\author{
Zina Trost ${ }^{1}$ and Thomas D. Parsons \\ Department of Psychology \\ University of North Texas
}

\begin{abstract}
The current review describes a novel virtual reality (VR) paradigm intended to optimize graded exposure treatment for pain-related fear and associated disability in chronic musculoskeletal pain, focusing on chronic low back pain. By combining an established treatment protocol with the innovative Kinect system by Microsoft, virtual reality graded exposure therapy (VRGET) addresses several central limitations of traditional exposure and VR approaches to pain/disability treatment. In addition to mitigating costs associated with traditional exposure protocols, the VRGET platform facilitates patient treatment engagement, provides real-time assessment of valuable outcome variables such as affective response and kinematic adaptation, and promotes generalizability of treatment gains across clinical and home environments. The current review provides an overview of current and future clinical directions for this new technology.
\end{abstract}

\section{Introduction}

Over the past several years, virtual reality (VR) technology has become incorporated into a number of interventions targeting physical (Holden, 2005), neurocognitive (Parsons, Rizzo, Rogers, \& York, 2009), and affective (Parsons \& Rizzo, 2008) conditions, including problematic pain (Keefe et al., 2012). To date, VR pain protocols have specifically focused on intervention in the acute pain context, relying on distraction properties of immersive VR platforms (Hoffman, Doctor, Patterson, Carrougher, \& Furness, 2000). In a recent topical review, Keefe et al. (2012) identified and discussed ways in which VR can be used either alone or in combination with other treatments not just for acute, but also for

${ }^{1}$ Correspondence concerning this article should be addressed to Zina Trost, Department of Psychology, University of North Texas, 1611 W. Mulberry Street, Denton, TX 76203, USA. E-mail: zina.trost@unt.edu 
persistent pain conditions. The authors concluded that although research on VR interventions for persistent pain is still in its infancy, the use of immersive virtual environments holds considerable promise. The current article describes a novel VR platform, focusing on the treatment of chronic low back pain (CLBP) using virtual reality graded exposure therapy (VRGET). Specifically, VRGET serves to combine an established treatment protocol for chronic pain-namely, graded exposure in vivo (GEXP) - with the innovative Kinect system by Microsoft. This platform extends traditional immersive contexts to a noninvasive and portable paradigm that allows for a patient's fully-body interaction with a gaming environment intended to reduce pain-related disability. Later, we provide a brief overview of the theoretical underpinning as well as empirical support for the exposure approach to disability reduction (Vlaeyen, Morley, Boersma, \& De Jong, 2012), including the limitations of existing GEXP formats. Subsequently, we discuss the traditional use and limitations of VR approaches to pain management and detail the manner in which VRGET aims to both optimize and address the limitations characterizing both traditional GEXP and VR methodologies.

\section{Theoretical Background: The Role of Fear and Avoidance in Disability}

Musculoskeletal pain is the dominant type of chronic pain affecting the world population, exerting an enormous impact on individuals, societies, and health care systems (Croft, 2010; World Health Organization, 2003). Among the musculoskeletal pain conditions, low back pain is the most common and often the most costly. The incidence of low back pain has reached epidemic proportions, affecting up to $84 \%$ of adults at least once in their lives (Dagenais, Caro, \& Haldeman, 2008). Most acute low back pain episodes are self-limited, with symptoms remitting within a few weeks and calling for little or no intervention. However, it is estimated that up to $10 \%$ of low back pain sufferers develop a chronic pain condition characterized by long-term pain and associated disability (Pengel, Herbert, Maher, \& Refshauge, 2003). The fear-avoidance (FA) model of low back pain represents a leading cognitive-behavioral account for the development and maintenance of pain and disability following acute back injury (Leeuw et al., 2007; Vlaeyen \& Linton, 2000). According to the FA model, fear that movement or physical activity will exacerbate pain or prompt (re)injury-also known as pain-related fear or kinesiophobia - is underscored by catastrophic appraisals of pain sensations (Grotle, Vollestad, \& Brox, 2006; Sieben, Vlaeyen, Tuerlinckx, \& Portegijs, 2002) that promote a self-perpetuating cycle of behavioral avoidance, hypervigilance, depression, and disuse, eventually resulting in functional disability. Pain-related fear emerges as a robust predictor of pain and disability at acute (George, Fritz, \& McNeil, 2006; Grotle et al., 2006; Sieben et al., 2002; Swinkels-Meewisse, Roelofs, Verbeek, Oostendorp, \& Vlaeyen, 2006), subacute (Poiraudeau, Rannou, Baron, et al., 2006; Poiraudeau, Rannou, 
Le Henanff, et al., 2006), and chronic (Crombez, Vlaeyen, Heuts, \& Lysens, 1999; Grotle, Vollestad, Veierod, \& Brox, 2004; McCracken, Gross, Aikens, \& Carnrike, 1996; Vlaeyen, Kole-Snijders, Rotteveel, \& Heuts, 1995) stages of back pain. Critically, individuals high in pain-related fear endorse beliefs that pain is a sign of serious tissue damage, as well as high motivation to avoid exertion or activity that might contribute to pain and therefore to perceived damage (George et al., 2006; Poiraudeau, Rannou, Baron, et al., 2006; Swinkels-Meewisse et al., 2006). Physical avoidance is conceptualized as a key mediating variable in the progression from acute to chronic pain (Grotle et al., 2006; Sieben et al., 2002).

Consistent with the FA model, research with individuals suffering from CLBP reliably links pain-related fear with escape from and avoidance of physical activity, resulting in impaired behavioral functioning (Vlaeyen et al., 1995). Among individuals with higher pain-related fear, avoidance is reflected in both limited physical exertion and behavioral strategies (such as excessively guarded movement patterns) adopted to ostensibly reduce pain/harm (Crombez et al., 1999; McCracken et al., 1996). While immediate avoidance of stressful activity is a natural response to protect damaged tissues, prolonged avoidance is known to detrimentally affect various physical/physiological systems (Poiraudeau, Rannou, Baron, et al., 2006). In this way, avoidance behavior serves to maintain disability, and paradoxically, increase the chance of further injury (Crombez et al., 1999; McCracken et al., 1996). Furthermore, because avoidance behavior occurs in anticipation of rather than in response to pain, opportunities to receive corrective feedback regarding erroneous catastrophic pain beliefs are limited (Leeuw et al., 2007).

\section{GEXP}

GEXP was developed as a cognitive-behavioral intervention intended to reduce pain-related fear, catastrophizing, and disability (Grotle et al., 2004; Martinez, Sanchez, Miro, Medina, \& Lami, 2011; Turner, Jensen, Warms, \& Cardenas, 2002; Vlaeyen et al., 1995). Exposure protocols are typically delivered in outpatient or inpatient settings, and involve the establishment of a hierarchy of avoided activities and gradual confrontation of these feared activities through "behavioral experiments" intended to correct erroneous pain beliefs (Grotle et al., 2004). These idiosyncratic fear hierarchies are associated with individuals' beliefs regarding the harm/injury potential of various physical activities. By successive gradual exposure to previously avoided activities, individuals are able to correct catastrophic misinterpretations of pain sensations and specific harm expectancies, leading to decreased levels of fear and functional improvements (Grotle et al., 2004; Heuts et al., 2004; Somers et al., 2009; Sullivan et al., 2009; Turner et al., 2002). A growing number of clinical studies and randomized clinical trials demonstrate the clinical effectiveness of GEXP in fearful CLBP adults 
(Boersma et al., 2004; Crombez, Eccleston, Van Damme, Vlaeyen, \& Karoly, 2012; de Jong et al., 2005; Leeuw et al., 2008; Linton et al., 2008; Vlaeyen, de Jong, Geilen, Heuts, \& van Breukelen, 2001, 2002; Woods \& Asmundson, 2008). In addition, a related line of experimental research supports the effects of GEXP, demonstrating that highly fearful individuals' appraisals of the pain/harm value of specific physical activities are amenable to modification upon exposure (Crombez et al., 2002; Goubert, Crombez, \& Danneels, 2005; Goubert, Crombez, \& Lysens, 2005; Goubert, Francken, Crombez, Vansteenwegen, \& Lysens, 2002; Trost, France, \& Thomas, 2008). ${ }^{1}$

Despite their demonstrated effectiveness, existing GEXP protocols are characterized by a number of significant limitations (Woods \& Asmundson, 2008). First, the patient works with a dedicated clinician and often a comprehensive rehabilitation team (Vlaeyen et al., 2012). In this way, GEXP protocols may be somewhat expensive and time consuming, relying on trained interventionists over an indefinite number of sessions (Vlaeyen et al., 2012). Another challenge acknowledged by GEXP developers is that of patient engagement; while empirically most effective, GEXP does not appear to be a patient-preferred treatment, and is characterized by a high dropout rate (George \& Robinson, 2010; Vlaeyen et al., 2012). It has been suggested that patient nonadherence is likely due to the anxiety-provoking nature of an intervention specifically designed to challenge fearful pain beliefs (Hadjistavropoulos \& Kowalyk, 2004).

Additionally, current GEXP protocols offer only marginal metrics for understanding and tracking important aspects of rehabilitative challenges and gains. For instance, although cognitive/emotional reappraisal of physical activity is conceptualized as a central mechanism in the treatment process, existing GEXP protocols are not able to systematically track patient-affective response (i.e., fear or anxiety) to progressive physical challenge (Vlaeyen et al., 2012). In addition, patients with high levels of pain-related fear demonstrate subtle behavioral adjustments - such as guarded movement patterns or "splinting" of back musculature - that may function as safety behaviors; thus, limiting actual exposure to the feared (movement) stimulus. As will be discussed later, subtle postural adjustments during behavioral performance may actually facilitate continued pain experience and prompt (re)injury (Thomas \& France, 2007). Current GEXP

\footnotetext{
${ }^{1}$ Although the current article focuses on CLBP as a model for examining the proposed VRGET intervention, pain-related fear has been shown to predict pain, disability, and rehabilitation outcomes across a number of traumatic, chronic, and progressive musculoskeletal disorders. These include conditions such as spinal cord injury (Turner et al., 2002), fibromyalgia (Martinez et al., 2011), and osteoarthritis (Heuts et al., 2004; Somers et al., 2009) as well as outcomes following medical and surgical interventions such as total knee replacement (Sullivan et al., 2009). The treatment approach described (GEXP) has likewise been successfully applied across a range of disabling pain conditions (Vlaeyen, Morley, Boersma, \& De Jong, 2012).
} 
protocols offer no means to assess or intervene on these more subtle (and possibly unconscious) forms of avoidance behavior. Finally, GEXP treatment gains show somewhat limited generalizability from the treatment clinic to the home environment, as well as limited transfer of learning across discrete physical activities (Crombez et al., 2002; Goubert, Crombez, \& Danneels, 2005; Goubert, Crombez, \& Lysens, 2005; Goubert et al., 2002; Trost et al., 2008).

In sum, a number of significant limitations suggest that existing GEXP interventions can be optimized in terms of delivery, metrics, and establishment of therapeutic change, which can be addressed using VR-based delivery. Later, we discuss the benefits and limitations of traditional uses of VR in pain management, and describe the development of a VR exposure-based intervention protocol, specifically, VRGET.

\section{VR as Pain Distraction}

To date, VR applications have been employed almost exclusively in the context of acutely painful experiences/interventions (e.g., wound dressing, dental pain; Hoffman et al., 2000; Keefe et al., 2012). These acute VR interventions have traditionally relied on head-mounted displays (HMDs) and are understood as distractors that reduce cognitive resources available for perception of and cognitive elaboration on nociceptive input. In turn, decreased attention available for conscious pain processing has been shown to result in patients reporting less pain experience on subjective questionnaires (see McCaul, Monson, \& Maki, 1992). Developers of HMD-mediated interventions suggest that HMD-delivered immersive VR can offer a level of distraction that goes beyond that found in simple forms of distraction (e.g., watching videos or playing video games; see Hoffman, Prothero, \& Wells, 1998). It is further argued that VR may improve analgesia through the reduction of visual cues associated with a painful procedure (Hoffman et al., 2000). In a recent systematic review of virtual environments designed for pain distraction, results suggested that the use of VR in adjunct with standard pharmacologic analgesics produces lower pain scores (during changes in wound dressing and physical therapy) than pharmacologic analgesics alone (Malloy \& Milling, 2010).

While VR interventions have demonstrated effectiveness in the acute pain context, from the perspective of FA theory and in terms of chronic pain, there are a number of issues with interventions that rely exclusively on distraction. First, experimental pain studies suggest that individuals characterized by high fear and catastrophizing may not benefit from distraction to the same extent as their low-fear counterparts (Campbell et al., 2010; Roelofs, Peters, van der Zijden, \& Vlaeyen, 2004). In addition, as noted earlier, fearful individuals may engage in safety-seeking behavior during exposure to feared stimuli, effectively diminishing the effect of exposure (McCracken \& Eccleston, 2003; Vlaeyen et al., 2012; 
Vowles, McCracken, \& Eccleston, 2007). In this way, distraction from the emotional and cognitive content of fear during exposure is conceptualized as avoidance that may disrupt the therapeutic mechanisms of GEXP. Recent GEXP theorizing has in fact advocated incorporating experiential treatment approaches (e.g., mindfulness) to facilitate engagement with and tolerance of unpleasant emotions, cognitions, and pain sensations (Vlaeyen et al., 2012; Vowles et al., 2007).

As noted, clinical evidence for VR has stemmed almost exclusively from acute pain interventions where immediate analgesia is the primary focus (Keefe et al., 2012). Conversely, for many CLBP patients, complete pain relief is not an option (McCracken, 1998), and patients who persist in "attempting to solve the problem of pain" show poorer outcomes (Eccleston \& Crombez, 2007; Trost, Vangronsveld, Linton, Quartana, \& Sullivan, 2012). Although increased physical and social engagement may naturally diminish pain experience through distraction processes (as more stimuli vie for an individual's attention), the goal of GEXP is to encourage patients to participate in valued life activities despite potentially ongoing pain experience. Specifically, GEXP aims to break the association between perception of pain and the appraisal of physical harm or damage. In this way, the primarily goal of GEXP is not pain amelioration (as that may be impossible), but functional restoration through behavioral engagement (Vlaeyen et al., 2012).

Finally, traditional applications of VR to pain distraction have involved simulations of environments far removed from those in which the patient must function (e.g., Snow World; Hoffman et al., 2000). While these virtual distraction environments often include an interactive component (e.g., shooting monsters; see Das, Grimmer, Sparnon, McRae, \& Thomas, 2005), they typically do not include activities consistent with patient real-life goals and activities of daily living. In contrast, exposure methodologies explicitly aim to situate the patient within contexts where treatment gains would be most useful (e.g., home or workplace).

Beyond Distraction: Treating Pain-Related Fear and Avoidance Behavior in Chronic Pain Using VR Graded Exposure Therapy

Promising applications of VR to persistent pain have been facilitated by conceptual and technological advances in VR interfaces. In contrast to HMDs, researchers have increasingly turned to projection systems that allow for wholebody tracking and body-state integration, thus providing the user with a less invasive experience of naturalistic interaction with a virtual environment. In turn, the virtual environment adapts in a fluid manner with head and body motion (Parsons \& Courtney, 2011). This trend has employed commercially available interfaces such as Microsoft Kinect, Nintendo Wii, Sony, and Eyetoy Konami 
Dance Dance Revolution, which present three-dimensional (3-D) graphic environments on flat screen monitors (Microsoft, 2011; Obdržálek et al., 2012). While such noninvasive VR systems involve a lower level of immersion, the interactive nature of the virtual environments allows the user's phenomenological experience to retain high potential for experience of digital content (i.e., via naturalistic rather than encumbered body actions in graphical simulations of real-world activities). Here it is important to note that the term "virtual reality" does not limit the researcher to a particular configuration of hardware and software. Instead, VR may be understood as a development of simulations that make use of various combinations of interaction devices and sensory display systems. Typically, the design of these systems is developed with consideration of balancing the level of immersiveness with the level of invasiveness. While historical users of VR have opted for highly immersive experiences using the more invasive HMDs, a new generation of simulation and serious gaming technologies serve as promising tools in many domains of assessment and therapy (Parsons, 2011; Parsons, McPherson, \& Interrante, 2013; Parsons \& Reinebold, 2012).

Drawing on these emerging technologies, researchers at the University of North Texas have developed an adaptive virtual environment for treatment of pain-related fear and avoidance behavior. This project brings together a team of researchers to incorporate cutting-edge pain assessment and intervention into a state-of-the-art interactive/adaptive VRGET protocol. The VR-based GEXP intervention is a free VR platform based on open-source software that can be downloaded via file transfer protocol (contact corresponding author). The software allows nonexpert users to adapt the content of pre-designed virtual environments to the specific needs of the clinical or experimental setting. The components needed for setting up the experimental platform are as follows: Windows Kinect; Core 2 duo 2.0 processor; OS Windows 7; NET FRAMEWORK 4.0; and Kinect for Windows SDK 1.8. In order to successfully use the Kinect system for VRGET, clinicians and researchers follow the manualized instructions (available via file transfer protocol upon request): (1) skeleton calibration; (2) skeleton/marker tracking; (3) motion data transformation; (4) game configuration; (5) game play; and (6) feedback and evaluation. After hardware setup, the user installs Microsoft Kinect for Windows skeleton tracker. To use the Microsoft skeleton tracker, the user must download and install the Microsoft Kinect for Windows SDK 1.8. Following the installation of drivers, a skeleton model is generated and adapted for each new patient. Using a separate tool, the "Calibration Assisted Patient Skeleton (CASP)," a calibrated skeleton model is automatically generated. To execute the algorithm the patient is instructed to perform some initial calibration movements. For an efficacious calibration, the patient is instructed to move the joints of interest to their full extent for about 15 seconds. For practical use with patients, the skeleton calibration assistant shows movement sequences. The VRGET platform is free to use and distribute for both 
commercial and noncommercial purposes. However, users must still abide by the licensing terms of any third party software installed for skeleton tracking.

As will be described later, VRGET serves to combine current approaches to GEXP with the innovative Kinect system by Microsoft. The Kinect is one of the most widely used whole-body trackers and has the ability to integrate body-state information into various simulations. The Kinect system uses image, audio, and depth sensors for movement detection, facial expression identification, and speech recognition. The Kinect's interactive technology allows users to interact with simulations using their own bodies as controls. An important advance in the Kinect technology is that, unlike previous attempts at gesture or movementbased controls, the patient is not encumbered by the need to wear accessories to enable the tracking of the patient's movements. In this way, the Kinect system represents an efficient tool for treatment of fear related to specific physical exertions (e.g., vacuuming, bending forward) as it inherently relies on (and captures) an individual's physical output.

The VRGET protocol has been designed to offer an adaptive virtual environment that can be explored by patients in their home under the supervision of a trained clinician. As with traditional GEXP, the individual tasks/components of each VRGET protocol would be guided by the idiosyncratic hierarchy of goals generated by the patient. Importantly, VRGET offers potential for automated monitoring and evaluation - described in detail later - that can be added to standard clinical evaluation methods. As a result, both clinicians and researchers will have access to a great deal more data for measuring exposure therapy progress. The monitoring and data-gathering potential of the VRGET system represents a major advance in detection of minor performance variations and affective changes that are not always sufficiently detectable by standard clinical scales that were constructed based on the human observer. By offering increased standardization of administration, increased accuracy of timing presentation and response latencies, ease of administration and data collection, and reliable and randomized presentation of stimuli for repeat administrations, VRGET offers the possibility for a new dimension of interactive exposure treatment, bolstering the clinical effectiveness of traditional GEXP by drawing on some of the inherent attributes of the emerging VR technology. Later, we discuss the major potential contributions of VRGET to traditional exposure approaches to pain-related fear and disability management.

\section{Engagement and Reinforcement}

As noted earlier, participant engagement and retention in treatment has been a major challenge for traditional GEXP interventions (Vlaeyen et al., 2012). It is important that exposure therapies be graded, motivating, and related to real-life functional activities, or they may fail to enlist active participation and/or 
decrements in avoidance behavior (Ferguson \& Trombly, 1997). At the most basic level, interacting within VRGET involves cognitive and motor activities that can engage a patient's attention (Parsons, Bowerly, Buckwalter, \& Rizzo, 2007; Parsons, Courtney, \& Dawson, 2013). Further, VRGET environments can be designed to be adaptive and offer increasingly difficult challenges with a level of gradation that cannot be facilitated by traditional GEXP (Parsons \& Courtney, 2011). In addition, VRGET can offer real-time participant feedback across a number of domains. A core component of traditional GEXP intervention is participants' recognition of their changing cognitive appraisals of physical activity; in addition to this traditional feedback, VRGET can offer feedback on noncognitive aspects of activity performance-for instance, performance velocity - that cannot be provided by a human interventionist, but that would facilitate recognition, reinforcement, and scaffolding of treatment gains.

Importantly, VRGET can integrate reinforcement contingencies for exposure to feared activities that are not part of traditional GEXP approaches. In addition to inherently novel stimuli and rehabilitative scenarios, VRGET can provide contingencies (e.g., elements drawn from gaming environments), that can add to the reward of activity performance, increasing patient motivation, and further solidifying treatment gains (Thornton et al., 2005). The motivational potential of VRGET is evidenced by the success of devices like Sony's PlayStation Move and Nintendo's Wii Remote Plus. While these "off-the-shelf" systems were developed for entertainment, a number of gaming engines have emerged that provide the monitoring and adaptation capabilities needed for use as rehabilitation applications (Bailey \& McInnis, 2011; Pirovano, Mainetti, Baud-Bovy, Lanzi, \& Borghese, 2012).

\section{Assessment of Emotional Responses to Exposure}

Fear is conceptualized as the key emotional component of avoidant behavior patterns. According to the FA model, an individual's fear is based upon the erroneous belief that pain signals physical damage, and thus any activity that exacerbates pain should be avoided (Vlaeyen \& Linton, 2000). As patients gradually confront feared activities, maladaptive pain beliefs are challenged and fear responses are extinguished (Vlaeyen et al., 2012). Prospective tracking of participants' fear responses would thus be central to GEXP intervention. However, current protocols rely only on participant retroactive self-report of fear in response to activity. Self-report is notoriously sensitive to a host of confounding influences, including a patient's desire to please their treatment provider.

A number of possibilities are available to address this limitation within the VRGET framework. First, the addition of minimally invasive psychophysiological sensors to the VRGET platform facilitates continuous objective measurement 
of the user's state, which can include measures of cognitive workload (Berka et al., 2007), varying stress levels (Fairclough \& Venables, 2006; Kobayashi, Yoshino, Takahashi, \& Nomura, 2007), task engagement (Pope, Bogart, \& Bartolome, 1995; Seery, Weisbuch, \& Blascovich, 2009), and arousal (Bradley \& Lang, 2000; Cuthbert, Bradley, \& Lang, 1996; Cuthbert, Schupp, Bradley, Birbaumer, \& Lang, 2000), among others. Technological advances in sensors, low-power integrated circuits, and wireless communication capabilities have allowed for the design of minimally invasive, miniature, lightweight, and low-cost wearable psychophysiological sensor nodes (Milenkovic, Otto, \& Jovanov, 2006) that are readily included in the VRGET protocol. A completely noninvasive approach to assessment of a patient's emotional response derives from Microsoft Kinect's capability to generate both RGB images and corresponding depth maps of scenes (Ren, Meng, Yuan, \& Zheng, 2011; Sempena \& Aryan, 2011). For instance, Burba, Bolas, Krum, and Suma (2012) described an exploratory study using Kinect to measure respiratory rate by measuring the visual expansion and contraction of the user's chest cavity during inhalation and exhalation. They also used Kinect to detect fidgeting behavior by measuring high-frequency vertical oscillations of the user's knees. Martinez and Stiefelhagen (2012) used a Kinectbased method to estimate the respiration rate of subjects from their chest movements. They fixed an infrared (IR) dot pattern that could be detected using the Kinect camera with a matching IR filter. The system was evaluated on nine subjects. These preliminary studies may be extended by isolating the chest cavity using more well-developed methods.

Researchers have also developed regularized maximum-likelihood deformable model fitting algorithms for 3-D face tracking with Kinect that allow for optimal use of Kinect's two-dimensional (2-D) color video and sampling of depth images at $30 \mathrm{fps}$ (Cai, Gallup, Zhang, \& Zhang, 2010). Mahmoud, Baltrušaitis, Robinson, and Riek (2011) used Kinect to develop a 3-D multimodal corpus of naturalistic complex mental states, consisting of 108 videos of 12 mental states. Likewise, Zhang et al. (2013) have used Kinect and the facial action coding system (FACS; Ekman, Friesen, \& Hager, 2002) to collect and establish a 3-D multimodal database for emotion recognition. The resulting database was concluded to be an effective FACS-based model with potential for effective facial-expression interpretation. Finally, the Microsoft Avatar Kinect uses facialexpression tracking and arm movements (through skeletal tracking) to allow researchers to control an avatar's head, facial expression, and arm movements. As a participant speaks, smiles, frowns, scowls, etc., her or his voice and facial expressions are recorded and can be enacted by the participant's avatar. Within the Avatar Kinect suite, researchers have access to 15 unique virtual environments that give context. Early research efforts are likewise underway to make use of Kinect to monitor emotional body language (e.g., Lala, Sutasinee, \& Toyoaki, 2011). 


\section{Kinematic Tracking of Movement Performance}

Kinect's skeletal tracking capabilities have been leveraged for rehabilitation (Chang, Chen, \& Huang, 2011), interactive storytelling, video games (Skalski, Lange, Tamborine, \& Shelton, 2007), and social robots (Ramey, Gonzalez-Pacheco, \& Salichs, 2011). These capabilities are of special importance in terms of interventions for pain-related fear, as increasing evidence suggests that pain-related fear may manifest as altered motor strategy in addition to more apparent avoidant behavior. Specifically, studies find that individuals with high pain-related fear may be particularly vulnerable to develop and maintain protective motor responses (e.g., reduced lumbar flexion) typically intended to splint/stiffen the spine initially after injury (Geisser, Haig, Wallbom, \& Wiggert, 2004; Thomas \& France, 2008; Trost, France, Sullivan, \& Thomas, 2012). Although these motor strategies initially enhance protection of damaged tissues (van Dieen, Cholewicki, \& Radebold, 2003; van Dieen, Selen, \& Cholewicki, 2003), continued restriction of motion and abnormal transfer of loads may predispose spinal structures to further damage, possibly contributing to recurrent or disabling pain experience (Hodges, 2011; MacDonald, Moseley, \& Hodges, 2009; van Dieen, Cholewicki, et al., 2003; van Dieen, Selen, et al., 2003). Continued motor guarding is hypothesized to owe to continued perception of threat associated with movement (Moseley \& Hodges, 2006).

While traditional GEXP protocols are not equipped to assess for these more insidious motor disturbances, Kinect's skeletal tracking capabilities allow the VRGET protocol to monitor/collect whole sets of dimensions necessary to describe each participant's height, poses, and movements. The building of a skeleton by Kinect requires a depth image of a participant's body and a sensor algorithm to develop an intermediate representation that maps the body (Shotton et al., 2011). While some parts used to make up the body-map representation are the participant's joints, others are links that connect the joints. These representational parts are coded, and algorithms recognize the codes to assign left and right to the sides of the represented body. The Kinect dataset recordings of patient movements can be captured using the OpenNI (http:// www.openni.org/) drivers/SDK and are OpenNI-encoded (.ONI). The OpenNI SDK provides a high-level skeleton tracking module that researchers may use to detect and track the captured patient poses and movements. The OpenNI tracking module produces the positions of 17 joints (head, neck, torso, left and right collars, left and right shoulders, left and right elbows, left and right wrists, left and right hip, left and right knees, and left and right feet), along with the corresponding tracking confidence. The OpenNI tracking module requires initial calibration relative to the patient so that the Kinect can further infer information related to the patient's height and body characteristics. Calibration 
of the Kinect skeleton requires the captured representation of the patient to remain in a fixed position or "calibration pose" for a few seconds.

With the Kinect's skeletal tracking, clinical researchers can represent a patient's body using a number of points (e.g., joints) representing various body parts: head, neck, shoulders, torso, arms, and legs. Each of these is represented by 3-D coordinates. The Kinect uses this information to determine the location and trajectory of all the 3-D parameters in real-time, which allows for fluid interactivity. For example, Alexiadis et al. (2011) evaluated the performance of a dancer via Kinect-based skeleton tracking. In their study, three different scores were calculated for a dancer's performance, which were subsequently combined to produce an overall score: (1) joint positions: a score for each joint was calculated by considering the modulus of the quaternionic correlation coefficient (CC) for each pair of joint position signals. A total score S1 was then computed as the weighted mean of the separate joint scores; (2) joint velocities: an overall score S2 was extracted based on the velocities of the joints (instead of their positions) by considering the quaternionic $\mathrm{CC}$ for the joint velocity signals; (3) 3-D flow error: for a given frame, the velocities of the joints were considered as 3-D motion (flow) vectors. Alexiadis et al. applied the work found in 2-D optical flow literature to 3 -D velocity vectors in homogenous coordinates and calculated the vectors' inner product to obtain a score for each joint (Barron, Feet, \& Beauchemin, 1994); and (4) combined score: the score was calculated as the weighted mean of the earlier three. This set of the three weights was selected using an optimization approach. In the same manner, the Kinect system can be calibrated to examine whether a CLBP patient is performing activities in a guarded manner, for instance by reducing velocity during certain physical moments or by maintaining rigid postural control during tasks that call for spinal motion.

\section{Generalizing Treatment Gains}

The generalization of treatment gains from the clinic to the home environment has been a major limitation for GEXP interventions (George \& Robinson, 2010). As with kinematic adaptations, individuals with high pain-related fear appear hesitant to abandon their appraisals of the harmful potential of physical activity. Specifically, learning may not transfer from one physical task to another, or to a similar task in a different environment (Bouton, 2000). For example, through traditional GEXP, a highly fearful patient may learn that bending to tie their shoe is safe, but may hesitate to perform similar flexion as part of a different task (e.g., vacuuming) (Crombez et al., 2002; Goubert, Crombez, \& Danneels, 2005; Goubert, Crombez, \& Lysens, 2005; Goubert et al., 2002). Thus, even with exposure, highly fearful participants are hesitant to change their fundamental belief that movement and pain are unsafe. 
Current GEXP protocols acknowledge this limitation and suggest a number of approaches to facilitate generalization of learning across activities and contexts. Specifically, clinicians are encouraged to provide homework to be carried out within patients' home environments and to incorporate multiple stimuli as part of exposure treatment (Vlaeyen et al., 2012). In addition, a recent study (Trost et al., 2008) demonstrated that patients can maintain learning effects across a progressively more difficult movement task (rather than across discrete movements). Thus, practicing behavior across different environments and creating a gradient of difficulty for discrete activities within an individuals' fear hierarchy are hypothesized to optimize treatment gains. As noted above, the flexibility of a simulated environment and maintenance of optimal engagement offered by the VRGET format further allows variations in grade/degree of exposure that may not be feasible within the constraints of traditional GEXP. That is, treatment "speed" can be optimally graded/scaffolded to participant comfort level, thereby facilitating success experiences. After initial orientation, treatment can be monitored and guided in part by the participant in their home environment.

\section{Conclusion}

VR technologies are increasingly being harnessed for therapeutic and rehabilitative goals both within the physical and psychological domain. Although most of these VR systems are not commercially available (or are extremely expensive when available), low-cost, accessible systems (e.g., Kinect, Nintendo Wii) are being tested for rehabilitation applications (Saposnik, Levin, \& Outcome Research Canada Working Group, 2011). By virtue of its intrinsically distracting properties, and with the aid of HMDs, VR has demonstrated considerable success in the arena of acute pain management (Keefe et al., 2012). However, as noted earlier, chronic musculoskeletal pain patients face a number of unique challenges that do not lend themselves to acute pain treatments, particularly among individuals with high pain-related fear. However, technological advances have resulted in new possibilities for combining VR with validated treatments for chronic musculoskeletal disability. As a hybrid of empirically validated clinical intervention and VR technology, we expect that VRGET can make major clinical and empirical contributions to the treatment and understanding of chronic musculoskeletal pain.

The flexibility and metrics offered by the Kinect interface are uniquely matched to enhance traditional exposure treatment. Specifically, the noninvasive and interactive format allows for uninhibited physical performance by the participant, which is key for exposure interventions targeting avoidance. By incorporating novel visual cues and gaming elements, VRGET can bolster participant engagement and adherence to an otherwise anxiety-provoking intervention. In this way, VRGET capitalizes on elements of distraction that are inherent to VR methodologies without sabotaging the exposure element necessary for treatment 
gains. Treatment gains can likewise be optimized by the ability of VR simulations to "scaffold" the difficulty of tasks across different contexts. This latter element can address the problem of treatment generalizability demonstrated by traditional GEXP. Finally, the advanced metrics offered by VRGET are useful in at least three ways. First, the system would allow for automated tracking of patient progress and data collection that can be distally examined by a clinician. Second, through addition of psychophysiological measurement or motion tracking, VRGET can examine participant affective response throughout the exposure process; this is particularly key as fear reduction is conceptualized as central to successful treatment. Third, VRGET would allow for tracking of motor responses that are not congruent with successful treatment engagement (e.g., guarding or bracing behavior); such monitoring is not possible within traditional clinical contexts. In summary, VRGET promises to be an affordable and highly accessible treatment option to reduce fear and disability in the context of chronic musculoskeletal pain. Empirical efforts will continue to refine the VRGET methodology toward optimal patient usability and wider dissemination.

\section{References}

Alexiadis, D. S., Kelly, P., Daras, P., O'Connor, N. E., Boubekeur, T., \& Moussa, M. B. (2011). Evaluating a dancer's performance via Kinect-based skeleton trackinga dancer's performance via kinect-based skeleton tracking. Paper presented at the Proceedings of the 19th ACM International Conference on Multimedia, New York, NY.

Bailey, B. W., \& McInnis, K. (2011). Energy cost of exergaming: A comparison of the energy cost of 6 forms of exergaming. Archives of Pediatrics and Adolescent Medicine, 165, 597-602.

Barron, L. J., Feet, D. J., \& Beauchemin, S. (1994). Performance of optical flow techniques. International Journal of Computer Vision, 12, 43-77.

Berka, C., Levendowski, D. J., Lumicao, M. N., Yau, A., Davis, G., Zivkovic, V. T., et al. (2007). EEG correlates of task engagement and mental workload in vigilance, learning, and memory tasks. Aviation, Space, and Environmental Medicine, 78 (5 Suppl), B231-B244.

Boersma, K., Linton, S., Overmeer, T., Jansson, M., Vlaeyen, J., \& de Jong, J. (2004). Lowering fear-avoidance and enhancing function through exposure in vivo. A multiple baseline study across six patients with back pain. Pain, 108, $8-16$.

Bouton, M. E. (2000). A learning theory perspective on lapse, relapse, and the maintenance of behavior change. Health Psychology, 19, 57-63.

Bradley, M. M., \& Lang, P. J. (2000). Affective reactions to acoustic stimuli. Psychophysiology, 37, 204-215. 
Burba, N., Bolas, M., Krum, D. M., \& Suma, E. A. (2012). Unobtrusive measurement of subtle nonverbal behaviors with the Microsoft Kinect. Virtual Reality Workshops (VR), IEEE, 1-4.

Cai, Q., Gallup, D., Zhang, C., \& Zhang, Z. (2010). 3D deformable face tracking with a commodity depth camera. Proceedings of the 11th European Conference on Computer Vision (ECCV).

Campbell, C. M., Witmer, K., Simango, M., Carteret, A., Loggia, M. L., Campbell, J. N., et al. (2010). Catastrophizing delays the analgesic effect of distraction. Pain, 149, 202-207.

Chang, Y. J., Chen, S. F., \& Huang, J. D. (2011). A Kinect-based system for physical rehabilitation: A pilot study for young adults with motor disabilities. Research in Developmental Disabilities, 32, 2566-2570.

Croft, P. (2010). The global occurence of chronic pain: An introduction. In P. Croft, F. M. Blyth \& D. van der Windt (Eds.), Chronic pain epidemiology: From aetiology to public health (pp. 9-18). Oxford: Oxford University Press.

Crombez, G., Eccleston, C., Van Damme, S., Vlaeyen, J. W. S., \& Karoly, P. (2012). Fear-avoidance model of chronic pain: The next generation. Clinical Journal of Pain, 28, 475-483.

Crombez, G., Eccleston, C., Vlaeyen, J. W., Vansteenwegen, D., Lysens, R., \& Eelen, P. (2002). Exposure to physical movements in low back pain patients: Restricted effects of generalization. Health Psychology, 21, 573-578.

Crombez, G., Vlaeyen, J. W., Heuts, P. H., \& Lysens, R. (1999). Pain-related fear is more disabling than pain itself: Evidence on the role of pain-related fear in chronic back pain disability. Pain, 80, 329-339.

Cuthbert, B. N., Bradley, M. M., \& Lang, P. J. (1996). Probing picture perception: Activation and emotion. Psychophysiology, 33, 103-111.

Cuthbert, B. N., Schupp, H. T., Bradley, M. M., Birbaumer, N., \& Lang, P. J. (2000). Brain potentials in affective picture processing: Covariation with autonomic arousal and affective report. Biological Psychology, 52, 95-111.

Dagenais, S., Caro, J., \& Haldeman, S. (2008). A systematic review of low back pain cost of illness studies in the United States and internationally. Spine Journal, 8, 8-20.

Das, D. A., Grimmer, K. A., Sparnon, A. L., McRae, S. E., \& Thomas, B. H. (2005). The efficacy of playing a virtual reality game in modulating pain for children with acute burn injuries: A randomized controlled trial. BMC Pediatrics, 5,1 .

de Jong, J. R., Vlaeyen, J. W., Onghena, P., Cuypers, C., den Hollander, M., \& Ruijgrok, J. (2005). Reduction of pain-related fear in complex regional pain syndrome type I: The application of graded exposure in vivo. Pain, 116, 264-275.

Eccleston, C., \& Crombez, G. (2007). Worry and chronic pain: A misdirected problem solving model. Pain, 132, 233-236. 
Ekman, P., Friesen, W. V., \& Hager, J. C. (2002). Facial action coding system: Research nexus. Salt Lake City, UT: Network Research Information.

Fairclough, S. H., \& Venables, L. (2006). Prediction of subjective states from psychophysiology: A multivariate approach. Biological Psychology, 71, 100110.

Ferguson, J. M., \& Trombly, C. A. (1997). The effect of added purpose and meaningful occupation on motor learning. American Journal of Occupational Therapy, 51, 508-515.

Geisser, M. E., Haig, A. J., Wallbom, A. S., \& Wiggert, E. A. (2004). Pain-related fear, lumbar flexion, and dynamic EMG among persons with chronic musculoskeletal low back pain. Clinical Journal of Pain, 20, 61-69.

George, S. Z., Fritz, J. M., \& McNeil, D. W. (2006). Fear-avoidance beliefs as measured by the fear-avoidance beliefs questionnaire: Change in fearavoidance beliefs questionnaire is predictive of change in self-report of disability and pain intensity for patients with acute low back pain. Clinical Journal of Pain, 22, 197-203.

George, S. Z., \& Robinson, M. E. (2010). Preference, expectation, and satisfaction in a clinical trial of behavioral interventions for acute and sub-acute low back pain. Journal of Pain, 11, 1074-1082.

Goubert, L., Crombez, G., \& Danneels, L. (2005). The reluctance to generalize corrective experiences in chronic low back pain patients: A questionnaire study of dysfunctional cognitions. Behaviour Research and Therapy, 43, 1055-1067.

Goubert, L., Crombez, G., \& Lysens, R. (2005). Effects of varied-stimulus exposure on overpredictions of pain and behavioural performance in low back pain patients. Behaviour Research and Therapy, 43, 1347-1361.

Goubert, L., Francken, G., Crombez, G., Vansteenwegen, D., \& Lysens, R. (2002). Exposure to physical movement in chronic back pain patients: No evidence for generalization across different movements. Behaviour Research and Therapy, 40, 415-429.

Grotle, M., Vollestad, N. K., \& Brox, J. I. (2006). Clinical course and impact of fear-avoidance beliefs in low back pain: Prospective cohort study of acute and chronic low back pain. Spine, 31, 1038-1046.

Grotle, M., Vollestad, N. K., Veierod, M. B., \& Brox, J. I. (2004). Fearavoidance beliefs and distress in relation to disability in acute and chronic low back pain. Pain, 112, 343-352.

Hadjistavropoulos, H. D., \& Kowalyk, K. M. (2004). Patient-therapist relationships among patients with pain-related fear. In G. J. Asmundson, J. Vlaeyen \& G. Crombez (Eds.), Understanding and treating fear of pain (pp. 237-266). Oxford: Oxford University Press.

Heuts, P. H., Vlaeyen, J. W., Roelofs, J., de Bie, R. A., Aretz, K., van Weel, C., et al. (2004). Pain-related fear and daily functioning in patients with osteoarthritis. Pain, 110, 228-235. 
Hodges, P. W. (2011). Pain and motor control: From the laboratory to rehabilitation. Journal of Electromyography and Kinesiology, 21, 220-228.

Hoffman, H. G., Doctor, J. N., Patterson, D. R., Carrougher, G. J., \& Furness, T. A., 3rd. (2000). Virtual reality as an adjunctive pain control during burn wound care in adolescent patients. Pain, 85, 305-309.

Hoffman, H. G., Prothero, J., \& Wells, M. (1998). Virtual chess: The role of meaning in the sensation of presence. International Journal of HumanComputer Interaction, 10, 251-263.

Holden, M. K. (2005). Virtual environments for motor rehabilitation: Review. Cyberpsychology and Behavior, 8, 187-211.

Keefe, F. J., Huling, D. A., Coggins, M. J., Keefe, D. F., Zachary Rosenthal, M., Herr, N. R., et al. (2012). Virtual reality for persistent pain: A new direction for behavioral pain management. Pain, 153, 2163-2166.

Kobayashi, N., Yoshino, A., Takahashi, Y., \& Nomura, S. (2007). Autonomic arousal in cognitive conflict resolution. Autonomic Neuroscience, 132, 70-75.

Lala, D., Sutasinee, T., \& Toyoaki, N. (2011). Towards a virtual environment for capturing behavior in cultural crowds. Digital Information Management (ICDIM), 310-315.

Leeuw, M., Goossens, M. E., Linton, S. J., Crombez, G., Boersma, K., \& Vlaeyen, J. W. (2007). The fear-avoidance model of musculoskeletal pain: Current state of scientific evidence. Journal of Behavioral Medicine, 30, 77-94.

Leeuw, M., Goossens, M. E. J. B., van Breukelen, G. J. P., de Jong, J. R., Heuts, P. H. T. G., Smeets, R. J. E. M., et al. (2008). Exposure in vivo versus operant graded activity in chronic low back pain patients: Results of a randomized controlled trial. Pain, 138, 192-207.

Linton, S. J., Boersma, K., Jansson, M., Overmeer, T., Lindblom, K., \& Vlaeyen, J. W. S. (2008). A randomized controlled trial of exposure in vivo for patients with spinal pain reporting fear of work-related activities. European Journal of Pain, 12, 722-730.

MacDonald, D., Moseley, G. L., \& Hodges, P. W. (2009). Why do some patients keep hurting their back? Evidence of ongoing back muscle dysfunction during remission from recurrent back pain. Pain, 142, 183-188.

Mahmoud, M., Baltrušaitis, T., Robinson, P., \& Riek, L. (2011). 3D corpus of spontaneous complex mental states. In Proceedings of the International Conference on Affective Computing and Intelligent Interaction (ACII 2011). Lecture Notes in Computer Science.

Malloy, K. M., \& Milling, L. S. (2010). The effectiveness of virtual reality distraction for pain reduction: A systematic review. Clinical Psychological Review, 30, 1011-1018.

Martinez, M., \& Stiefelhagen, R. (2012). Breath rate monitoring during sleep using near-IR imagery and PCA. International Conference on Pattern Recognition (ICPR 2012), Skukuba. 
Martinez, M. P., Sanchez, A. I., Miro, E., Medina, A., \& Lami, M. J. (2011). The relationship between the fear-avoidance model of pain and personality traits in fibromyalgia patients. Journal of Clinical Psychology in Medical Settings, 18, 380-391.

McCaul, K. D., Monson, N., \& Maki, R. H. (1992). Does distraction reduce pain-produced distress among college students? Health Psychology, 11, 210217.

McCracken, L. A., \& Eccleston, C. (2003). Coping or acceptance: What to do about chronic pain? Pain, 105, 197-204.

McCracken, L. M. (1998). Learning to live with the pain: Acceptance of pain predicts adjustment in persons with chronic pain. Pain, 74, 21-27.

McCracken, L. M., Gross, R. T., Aikens, J., \& Carnrike, C. L. Jr. (1996). The assessment of anxiety and fear in persons with chronic pain: A comparison of instruments. Behaviour Research and Therapy, 34, 927-933.

Microsoft. (2011). Kinect full body interaction. Retrieved 9. 1. 2013, from http:// www.xbox.com/kinect

Milenkovic, A., Otto, C., \& Jovanov, E. (2006). Wireless sensor network for personal health monitoring: Issues and an implementation. Computer Communications, 29, 2521-2533.

Moseley, G. L., \& Hodges, P. W. (2006). Reduced variability of postural strategy prevents normalization of motor changes induced by back pain: A risk factor for chronic trouble? Behavioral Neuroscience, 120, 474-476.

Obdržálek, Š., Kurillo, G., Ofli, F., Bajcsy, R., Seto, E., Jimison, H., et al. (2012). Accuracy and robustness of Kinect pose estimation in the context of coaching of elderly population. Presented at the 34th Annual International Conference of the IEEE Engineering in Medicine and Biology Society, San Diego, CA.

Parsons, T. D. (2011). Neuropsychological assessment using virtual environments: Enhanced assessment technology for improved ecological validity. In S. Brahnam (Ed.), Advanced computational intelligence paradigms in healthcare (pp. 271-289). Germany: Springer-Verlag.

Parsons, T. D., Bowerly, T., Buckwalter, J. G., \& Rizzo, A. A. (2007). A controlled clinical comparison of attention performance in children with ADHD in a virtual reality classroom compared to standard neuropsychological methods. Child Neuropsychology, 13, 363-381.

Parsons, T. D., \& Courtney, C. (2011). Neurocognitive and psychophysiological interfaces for adaptive virtual environments. In C. R. A. T. Ziefle (Ed.), Human centered design of e-health technologies (pp. 208-233). Hershey, PA: IGI Global.

Parsons, T. D., Courtney, C., \& Dawson, M. (2013). Virtual reality Stroop task for assessment of supervisory attentional processing. Journal of Clinical and Experimental Neuropsychology, 35, 812-826. 
Parsons, T. D., McPherson, S., \& Interrante, V. (2013). Enhancing neurocognitive assessment using immersive virtual reality. Proceedings of the 17 th IEEE Virtual Reality Conference: Workshop on Virtual and Augmented Assistive Technology (VAAT).

Parsons, T. D., \& Reinebold, J. (2012). Adaptive virtual environments for neuropsychological assessment in serious games. IEEE Transactions on Consumer Electronics, 58, 197-204.

Parsons, T. D., \& Rizzo, A. A. (2008). Affective outcomes of virtual reality exposure therapy for anxiety and specific phobias: A meta-analysis. Journal of Behavioral Therapy and Experimental Psychiatry, 39, 250-261.

Parsons, T. D., Rizzo, A. A., Rogers, S. A., \& York, P. (2009). Virtual reality in pediatric rehabilitation: A review. Developmental Neurorehabilitation, 12, $224-238$.

Pengel, L. H., Herbert, R. D., Maher, C. G., \& Refshauge, K. M. (2003). Acute low back pain: Systematic review of its prognosis. BMJ (Clinical Research Ed.), 327, 323.

Pirovano, M., Mainetti, R., Baud-Bovy, G., Lanzi, P. L., \& Borghese, N. A. (2012). Self-adaptive games for rehabilitation at home. Proceedings of IEEE Conference on Computational Intelligence and Games, Granada.

Poiraudeau, S., Rannou, F., Baron, G., Le Henanff, A., Coudeyre, E., Rozenberg, S., et al. (2006). Fear-avoidance beliefs about back pain in patients with subacute low back pain. Pain, 124, 305-311.

Poiraudeau, S., Rannou, F., Le Henanff, A., Coudeyre, E., Rozenberg, S., Huas, D., et al. (2006). Outcome of subacute low back pain: Influence of patients' and rheumatologists' characteristics. Rheumatology (Oxford, England), 45, $718-723$.

Pope, A. T., Bogart, E. H., \& Bartolome, D. S. (1995). Biocybernetic system evaluates indices of operator engagement in automated task. Biological Psychology, 40, 187-195.

Ramey, A., Gonzalez-Pacheco, V., \& Salichs, M. A. (2011). Integration of a low-cost $R G B-D$ sensor in a social robot for gesture recognition. Paper presented at the 6th ACM/IEEE International Conference on Human-Robot Interaction (HRI), Lausanne.

Ren, Z., Meng, J., Yuan, J., \& Zheng, Z. (2011). Robust hand gesture recognition with Kinect sensor. ACM International Conference on Multimedia, Scottsdale, AZ.

Roelofs, J., Peters, M. L., van der Zijden, M., \& Vlaeyen, J. W. (2004). Does fear of pain moderate the effects of sensory focusing and distraction on cold pressor pain in pain-free individuals? Journal of Pain, 5, 250-256.

Saposnik, G., Levin, M., \& Outcome Research Canada Working Group. (2011). Virtual reality in stroke rehabilitation: A meta-analysis and implications for clinicians. Stroke, 42, 1380-1386. 
Seery, M. D., Weisbuch, M., \& Blascovich, J. (2009). Something to gain, something to lose: The cardiovascular consequences of outcome framing. International Journal of Psychophysiology, 73, 308-312.

Sempena, N. U. M., \& Aryan, P. R. (2011). Human action recognition using dynamic time warping. International Conference on Electrical Engineering and Informatics, Bandung.

Shotton, J., Fitzgibbon, A., Cook, M., Sharp, T., Finoccio, M., Moore, R., et al. (2011). Real-time human pose recognition in parts from single depth images. Paper presented at the IEEE Conference on Computer Vision and Pattern Recognition, Colorado Springs, CO USA.

Sieben, J. M., Vlaeyen, J. W., Tuerlinckx, S., \& Portegijs, P. J. (2002). Painrelated fear in acute low back pain: The first two weeks of a new episode. European Journal of Pain, 6, 229-237.

Skalski, P., Lange, R. L., Tamborine, R., \& Shelton, A. K. (2007). Mapping the road to fun: Natural video game controllers, presence, and game enjoyment. Paper presented at the Annual meeting of the International Communication Association, San Francisco, CA.

Somers, T. J., Keefe, F. J., Pells, J. J., Dixon, K. E., Waters, S. J., Riordan, P. A., et al. (2009). Pain catastrophizing and pain-related fear in osteoarthritis patients: Relationships to pain and disability. Journal of Pain and Symptom Management, 37, 863-872.

Sullivan, M., Tanzer, M., Stanish, W., Fallaha, M., Keefe, F. J., Simmonds, M., et al. (2009). Psychological determinants of problematic outcomes following total knee arthroplasty. Pain, 143, 123-129.

Swinkels-Meewisse, I. E., Roelofs, J., Verbeek, A. L., Oostendorp, R. A., \& Vlaeyen, J. W. (2006). Fear-avoidance beliefs, disability, and participation in workers and non-workers with acute low back pain. Clinical Journal of Pain, $22,45-54$.

Thomas, J. S., \& France, C. R. (2007). Pain-related fear is associated with avoidance of spinal motion during recovery from low back pain. Spine, 32, E460-E466.

Thomas, J. S., \& France, C. R. (2008). The relationship between pain-related fear and lumbar flexion during natural recovery from low back pain. European Spine Journal, 17, 97-103.

Thornton, M., Marshall, S., McComas, J., Finestone, H., McCormick, A., \& Sveistrup, H. (2005). Benefits of activity and virtual reality based balance exercise programmes for adults with traumatic brain injury: Perceptions of participants and their caregivers. Brain Injury, 19, 9891000.

Trost, Z., France, C. R., Sullivan, M. J., \& Thomas, J. S. (2012). Pain-related fear predicts reduced spinal motion following experimental back injury. Pain, 153, 1015-1021. 
Trost, Z., France, C. R., \& Thomas, J. S. (2008). Exposure to movement in chronic back pain: Evidence of successful generalization across a reaching task. Pain, 137, 26-33.

Trost, Z., Vangronsveld, K., Linton, S. J., Quartana, P. J., \& Sullivan, M. J. (2012). Cognitive dimensions of anger in chronic pain. Pain, 153, 515-517.

Turner, J. A., Jensen, M. P., Warms, C. A., \& Cardenas, D. D. (2002). Catastrophizing is associated with pain intensity, psychological distress, and pain-related disability among individuals with chronic pain after spinal cord injury. Pain, 98, 127-134.

van Dieen, J. H., Cholewicki, J., \& Radebold, A. (2003). Trunk muscle recruitment patterns in patients with low back pain enhance the stability of the lumbar spine. Spine (Phila Pa 1976), 28, 834-841.

van Dieen, J. H., Selen, L. P., \& Cholewicki, J. (2003). Trunk muscle activation in low-back pain patients, an analysis of the literature. Journal of Electromyography and Kinesiology, 13, 333-351.

Vlaeyen, J., Morley, S., Boersma, K., \& De Jong, J. (2012). Pain-related fear: Exposure-based treatment for chronic pain. Seattle, WA: IASP Press.

Vlaeyen, J. W., de Jong, J., Geilen, M., Heuts, P. H., \& van Breukelen, G. (2001). Graded exposure in vivo in the treatment of pain-related fear: A replicated single-case experimental design in four patients with chronic low back pain. Behaviour Research and Therapy, 39, 151-166.

Vlaeyen, J. W., de Jong, J., Geilen, M., Heuts, P. H., \& van Breukelen, G. (2002). The treatment of fear of movement/(re)injury in chronic low back pain: Further evidence on the effectiveness of exposure in vivo. Clinical Journal of Pain, 18, 251-261.

Vlaeyen, J. W., Kole-Snijders, A. M. J., Rotteveel, A. M., \& Heuts, P. H. (1995). The role of fear of movement/(re)injury in pain disability. Journal of Occupational Rehabilitation, 5, 235-252.

Vlaeyen, J. W., \& Linton, S. J. (2000). Fear-avoidance and its consequences in chronic musculoskeletal pain: A state of the art. Pain, 85, 317-332.

Vowles, K. E., McCracken, L. M., \& Eccleston, C. (2007). Processes of change in treatment for chronic pain: The contributions of pain, acceptance, and catastrophizing. European Journal of Pain, 11, 779-787.

Woods, M. P., \& Asmundson, G. J. G. (2008). Evaluating the efficacy of graded in vivo exposure for the treatment of fear in patients with chronic back pain: A randomized controlled clinical trial. Pain, 136, 271-280.

World Health Organization. (2003). The burden of musculoskeletal conditions at the start of the new millennium: Report of a WHO scientific group. Geneva: World Health Organization.

Zhang, Y., Zhang, L., \& Hossain, A. (2012). Multimodal Intelligent Affect Detection with Kinect. Proceedings of the 12th International Conference on Autonomous Agents and Multiagent Systems, St. Paul, Minnesota, USA 